\title{
Ensayo de respuesta térmica de suelo de un serpentín horizontal
}

Ing. Valentín Stefanini ${ }^{1}$; Ing. Daniel Nazario ${ }^{2}$

\section{RESUMEN}

Este trabajo se realizó con el objeto de describir los resultados que se obtuvieron a partir de un Test de Respuestas Térmicas (TRT), en el cual se determinaron los valores de la conductividad térmica efectiva del subsuelo $\lambda$ eff, y la resistencia térmica $\mathrm{Rb}$, correspondiente a un sistema horizontal de intercambiador particular de calor, realizado en la provincia de San Luis-Argentina.

Este ensayo se basó en el modelo de la fuente de línea infinita de kelvin (ILS), de transferencia de calor por conducción térmica. Cuya solución se obtuvo por el método grafico de la pendiente. A partir de ellos es posible diseñar un sistema de calefacción y refrigeración en edificios de bombas de calor acopladas al subsuelo, con un eventual ahorro energético y por ende disminución de la producción de dióxido de carbono.

Palabras clave: TRT, intercambiador horizontal, método pendiente.

\section{ABSTRACT}

This work was carried out in order to describe the results that were obtained from a
Thermal Response Test (TRT), in which the values of the effective thermal conductivity of the subsoil $\lambda$ eff, and the thermal resistance $R b$, corresponding to a horizontal system of particular heat exchanger, made in the province of San Luis-Argentina.

This test was based on the infnite line kelvin source (ILS) model, heat transfer by thermal conduction. Whose solution was obtained by the graphical method of the slope. From them it is possible to design a heating and cooling system in buildings of heat pumps coupled to the subsoil, with an eventual energy saving and therefore a decrease in the production of carbon dioxide.

\section{INTRODUCCIÓN:}

El objetivo de este trabajo consiste en explorar y describir los resultados que se obtuvieron a partir de un Test de Respuestas Térmicas (TRT), en el cual se determinaron los valores de la conductividad térmica efectiva del subsuelo $\lambda$ eff, y la resistencia térmica $\mathrm{Rb}$, correspondiente a un sistema horizontal de intercambiador particular de calor.

\footnotetext{
${ }^{1}$ Ingeniero Valentín Stefanini, Universidad Nacional de Villa Mercedes-Argentina. stefanini28@hotmail.com.ar

${ }^{2}$ Ingeniero Daniel Nazario. Universidad Nacional de Villa Mercedes-Argentina. elmundial@hotmail.com
} 
Para ello se realiza el ensayo a mediados del mes de abril 2013, correspondiente a la estación de otoño en el hemisferio sur.

Los datos obtenidos se cargan en una planilla de cálculo y se los grafica con un diagrama de dispersión, luego a la nube de puntos se los linealiza obteniendo la recta de regresión lineal con su ecuación y el error medio cuadrático.

A partir de la recta de regresión línea se obtiene el valor de $\lambda$ efec, $y$ luego se procede a calcular el valor de la resistencia térmica entre el fluido y la pared del pozo denominada $\mathrm{Rb}\left({ }^{\circ} \mathrm{K} /(\mathrm{W} / \mathrm{m})\right)$.

\section{METODOLOGÍA}

Para la realización del experimento se utiliza los aportes teóricos de Eklöf $\mathrm{C}$, Gehlin S. (1996), Ingersoll, L.R. and Plass, H.J. 1948 y Carslaw and Jaeger; además se cuenta con los laboratorios de ensayo de la UNSL.

El experimento que se realizó a partir del ensayo de un intercambiador, es de corte cuantitativo-descriptivo, ya que permite determinar los parámetros del subsuelo, a partir de dichos parámetros se puede diseñar un sistema de bomba de calor, para un ahorro sustancial de energía eléctrica para climatización y como consecuencia menor generación de dióxido de carbono.

\section{MATERIALES}

Se utilizó una excavación para la construcción de una piscina de 4,5 $\mathrm{m}$ de largo por 2,3 $\mathrm{m}$ de ancho y de 1,5 $\mathrm{m}$ de profundidad sobre un piso de $7,5 \mathrm{~cm}$ de hormigón ya realizado, se colocó un serpentín de caño
PEAD $\Phi$ 3/4" K6 apto para conducciones de agua, se lo sujetó a una malla sima rellenándolo con otra capa de hormigón de 7,5 cm de espesor.

Así, el intercambiador quedó entre un bloque de $15 \mathrm{~cm}$ de hormigón sin ningún tipo de aditivo, como la bentonita. Luego se construyó un tanque de expansión y se lo aisló térmicamente, se colocó una bomba de circulación y posteriormente se instalaron los instrumentos de medida consistente en varios termómetros, dos higrómetros y un caudalímetro mecánico. La perturbación térmica se realizó con una resistencia eléctrica colocada sobre el agua del tanque con su respectiva conexión, también se determinó la potencia eléctrica mediante las mediciones de corriente y de tensión.

Los datos recolectados se los cagaron en una planilla de cálculo donde se los procesaron, determinando la recta de regresión lineal la cual nos conduce al coeficiente de conductividad térmica de subsuelo.

\section{DESARROLLO}

El objetivo del presente trabajo es realiza un test de respuesta térmicas para un intercambiador de características diferentes, ya que se lo adaptó a una excavación existente, por lo cual su diseño es horizontal y asimétrico, el mismo fue construido en San Luis, Argentina.

El ensayo se basó en el modelo de la fuente de línea infinita de Kelvin (LSM), plasmado en la tesis de Eklöf C, Gehlin S. (1996). La solución del modelo planteado se realizó por el método de la pendiente, determinando el coeficiente de conductivi- 
dad efectivo de subsuelo $\lambda$ eff, y la resistencia térmica de suelo $\mathrm{Rb}$

Los test de respuesta térmica convencionales se los realiza para intercambiadores vertical enterrado en un pozo individual, por el cual se le hace circular un fluido caloportador, al cual se lo perturba con una tasa de inyección de calor constante. En el modelo teórico, no se considerada la afectación de los pozos cercanos y se supone que la taza de inyección es constante.

Se denomina gradiente geotérmico al aumento de temperatura del subsuelo con el aumento de profundidad. Si el gradiente geotérmico no varía, entonces las condiciones de subsuelo se consideran estacionarias.

Ahora, cuando se inyecta calor en un pozo de sondeo, el campo de temperatura comienza a variar y cuanto más calor se inyecte, más caliente se vuelve el suelo, por lo cual la temperatura de subsuelo no perturbado se encontrará a una distancia mayor del pozo. Si el calor inyectado es constante, el campo de temperatura será nuevamente estacionario, pero tardará como mínimo de 20 a 25 años.

En esta situación, se genera un proceso térmico que puede analizarse en tres estadios:

PROCESO TRANSITORIO cuando se registra un aumento de la temperatura del subsuelo, PROCESO ESTACIONARIO cuando ya no se registra el aumento de temperatura y la velocidad de transferencia de calor del subsuelo al medio ambiente se iguala a la velocidad de inyección de calor al mismo, y UN PULSO cuando se produce una variación del calor de inyección y se su- perpone al proceso estacionario.

El pulso no conduce a un estado estacionario ya que el mismo es limitado en el tiempo, y se superpone a la temperatura estacionaria media Tr. Mientras que un proceso transitorio el aumento de temperatura es superpuesto a la temperatura de subsuelo no perturbado Tsur. Esto diferencia al pulso del proceso transitorio.

La ecuación fundamental de conducción del calor nos muestra que la variación de la temperatura depende de la difusividad ' $\alpha$ '.

$$
\frac{\partial^{2} T}{\partial r^{2}}+\frac{\partial^{2} T}{\partial y^{2}}+\frac{\partial^{2} T}{\partial z^{2}}=\frac{1}{\alpha} \cdot \frac{\partial T}{\partial t}
$$

Entonces la temperatura T, en un punto de coordenadas $(X, Y, Z)$, está determinada por el tiempo ' $t$ ', y la difusividad ' $\alpha$ '.

Esta difusividad ' $\alpha$ ' dada en [m2/seg] depende de las propiedades del material y demuestra si el material es buen o mal conductor térmico. A mayor valor del parámetro ' $\alpha$ ' el material es mejor conductor del calor. En condiciones estacionarias, la capacidad calorífica pierde importancia, como así también la derivada temporal, por lo que la ecuación de conducción de calor puede ser representada por la ecuación de Laplace.

$$
\frac{\partial^{2} T}{\partial x^{2}}+\frac{\partial^{2} T}{\partial y^{2}}+\frac{\partial^{2} T}{\partial z^{2}}=0
$$

Durante el proceso transitorio se obtiene:

$$
T_{r}(t)=T_{s u r}+T_{r q}(t)
$$


Durante el proceso de un pulso:

$$
T_{r}(t)=T_{r}+T_{r q}(t)
$$

Para el modelo matemático del test de respuesta térmica, se considerará sólo el proceso transitorio, ya que la superposición del pulso no es de importancia.

La relación entre la temperatura del subsuelo no perturbado, Tsur, y la temperatura del fluido caloportador Tf., se da por una resistencia térmica $\mathrm{Rb}\left[{ }^{\circ} \mathrm{K}(\mathrm{W} / \mathrm{m})\right]$, y resulta ser la resistencia entre el fluido portador de calor y la pared del pozo, y se la define como:

$$
T_{r}-T_{\text {sar }}=R_{b} \cdot q
$$

Cuando se inyecta calor al subsuelo, comienza un proceso transitorio, por lo que se establece una relación entre la temperatura de suelo no perturbado Tsur y la temperatura del fluido portador de calor Tf. la cual está dada según, Ingersoll, L.R. and Plass, H.J. 1948 y Carslaw and Jaeger, por:

$$
T_{f}=\frac{Q}{4 \pi \lambda H}\left(\ln \left(\frac{4 a t}{r_{0}^{2}}\right)-\gamma\right)+\frac{Q R_{t}}{H}+T_{s a r}
$$

Expresión valida a partir de un determinado tiempo:

$$
t \geq \frac{5 r_{0}^{2}}{\alpha} \quad t_{b}=\frac{\gamma H^{2}}{16 \alpha}[\mathrm{seg}]
$$

Después de un cierto tiempo de varias décadas " $t b$ ", el proceso transitorio culmina y da comienzo a un proceso estacionario, descripto por la siguiente ecuación:

$$
T_{f}-T_{s u r}=\frac{Q}{H}\left[\frac{1}{2 \pi \lambda} \ln \left(\frac{H}{2 r_{0}}\right)+R_{b}\right]
$$

\section{Realización del ensayo:}

En el tanque de expansión se colocó una solución de 100 litros de agua con 2 litros de glicol. La perturbación térmica se realizó con una resistencia eléctrica.

El ensayo se comienzo el día 19/04/2013 "sin perturbación térmica", para determinar la temperatura de suelo no perturbado. Esta sé la obtuvo como el promedio de la temperatura de salida del intercambiador, la cual fue Tsur $=21,65^{\circ} \mathrm{C}$. Este ensayo sin perturbación térmica culminó el día 20/04/2013, con la toma de datos realizada de forma aislada.

El día 20/04/2013, se comenzó (TRT) "con perturbación". La duración del mismo fue de 72 hs, y culminó el día 23/04/2013. La toma de datos se realizó de forma manual cada 10 minutos, con una inyección de 818,9 watts de potencia media y con un caudal circulante de 0,00042 (m3/seg).

La figura 2 nos muestra la evolución de la temperatura media del fluido portador de calor Tf, en función del $\ln (\mathrm{t})$ en horas, desde el momento en que se comienza a inyectarle calor hasta la culminación del test, asimismo se observa la recta de regresión lineal, con su ecuación y el error medio cuadrático.

\section{EL CRITERIO DEL TIEMPO}

Para este tipo de ensayos se ha establecido en un criterio de tiempo, donde los datos obtenidos por debajo del mismo no son considerados, para este ensayo resulto de 9 horas, 


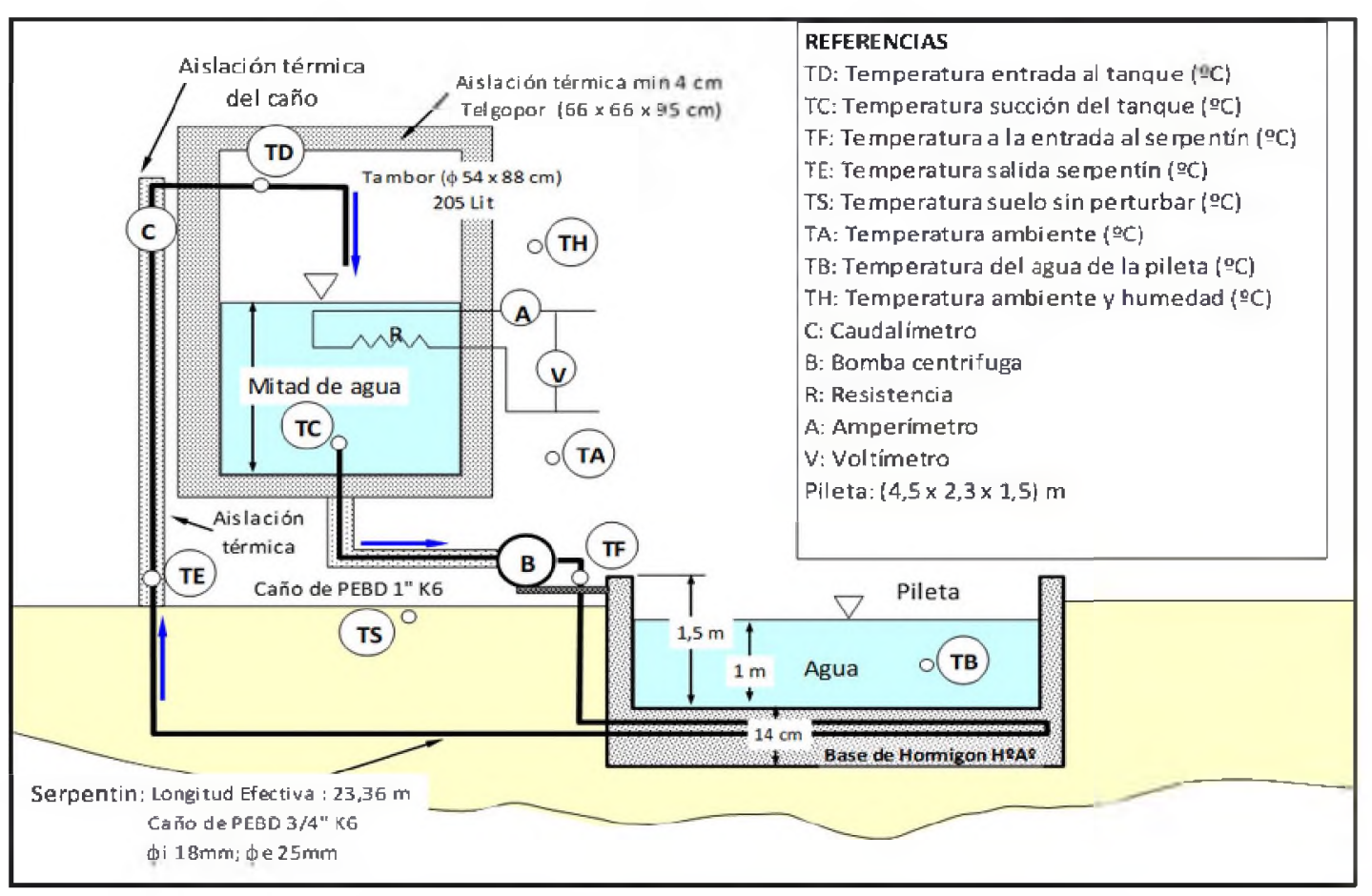

Figura 1: Esquema del dispositivo de medición montado en San Luís - Argentina.

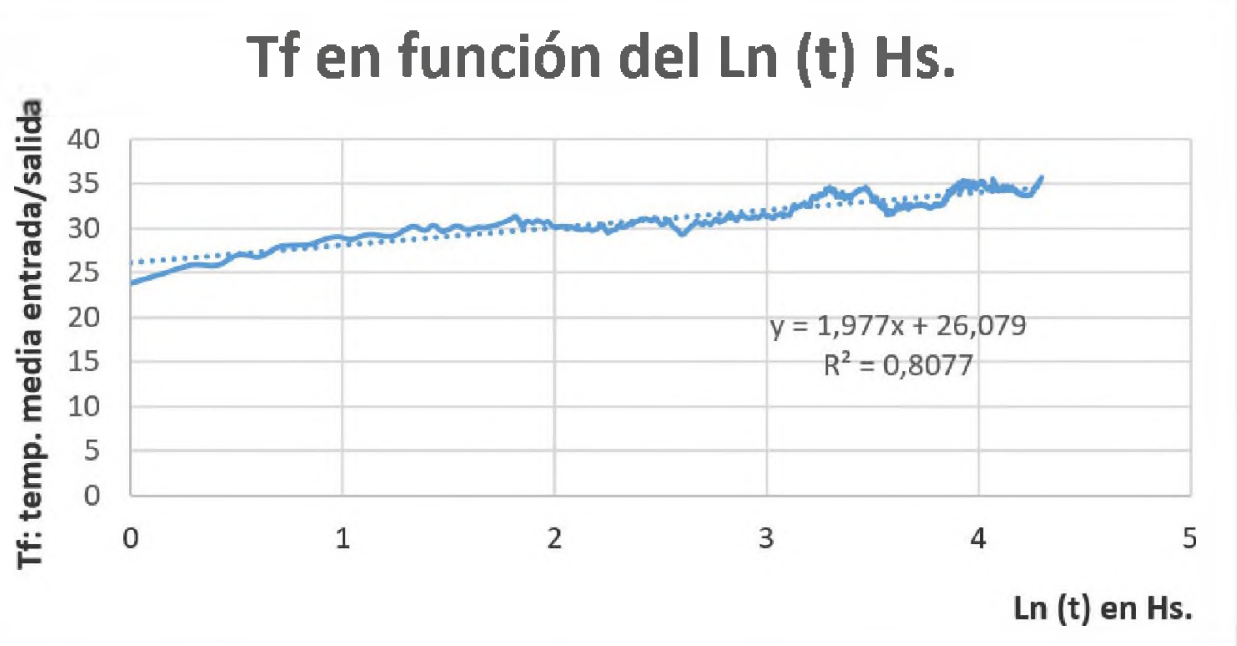

Figura 2: Temperaturas media entradalsalida en función del $\ln (t)$

a partir de este tiempo se evalúa la evolución de la temperatura media del fluido en función del logaritmo natural del tiempo, mostrado en la figura 3.

$$
t \geq \frac{5 r_{R}^{2}}{a} \quad t=\frac{5 \cdot 0,075^{2}}{0,91 \times 10^{-6}}=30907 \mathrm{seg}
$$




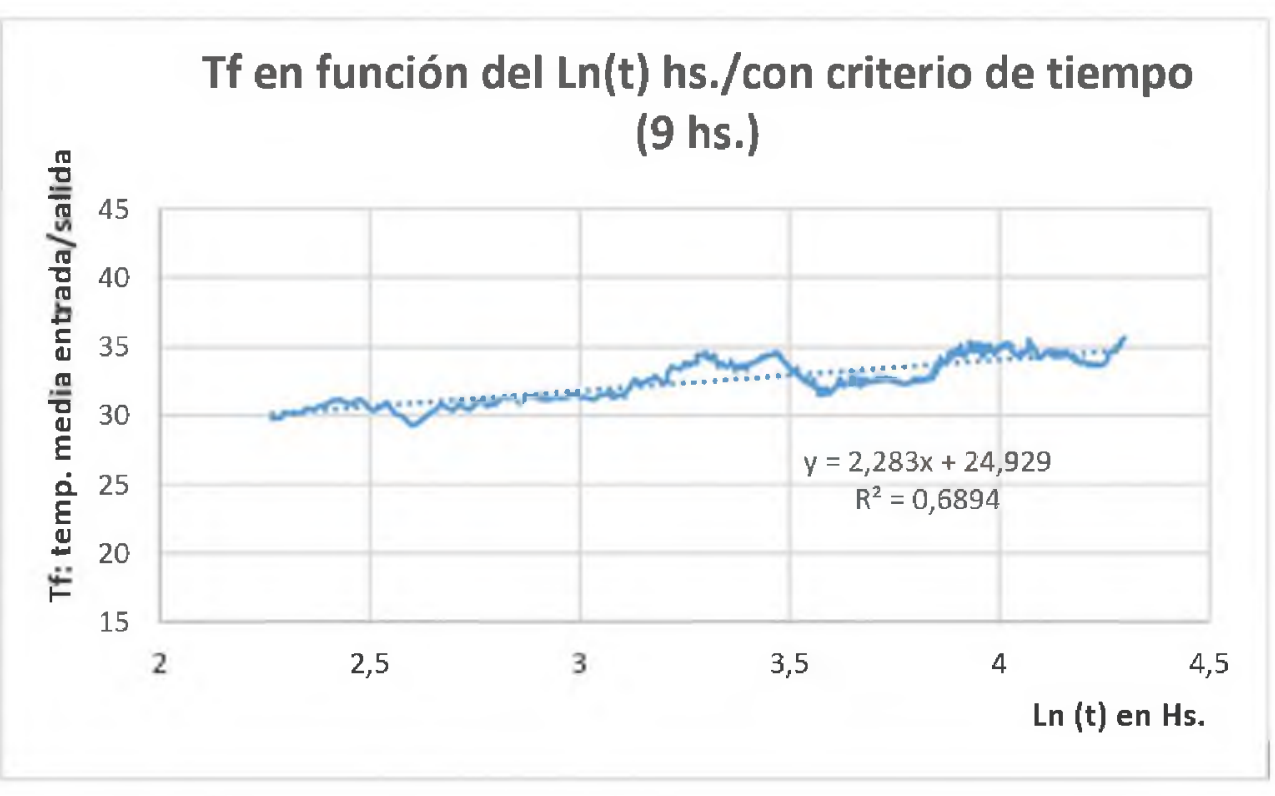

Figura 3: Evolución de la temperatura media entradalsalida en función del $\ln (t)$

en bs con criterio de tiempo

Para determinar el coeficiente de conductividad térmico efectivo por medio del método de la pendiente, el punto de partida es la ecuación de la recta de regresión lineal, aproximándolo por la siguiente ecuación:

$$
T_{f}=k \ln (t)+m \quad k=\frac{Q}{4 \pi \lambda H} \ln (t) \quad \lambda=\frac{Q}{4 \pi k H} \ln (t)
$$

$\lambda_{\text {eff:: }}$ para el ensayo total fue del orden de: $1,42 \mathrm{~W} / \mathrm{m}^{* 0} \mathrm{~K}$, para pendiente $\mathrm{K}=1,97$

$\lambda_{\text {eff:: }}$ aplicando el criterio de tiempo resulto: $1,23 \mathrm{~W} / \mathrm{m}^{* \circ} \mathrm{K}$, para pendiente $\mathrm{K}=2,28$ 


\section{DETERMINACIÓN DE LA RESISTENCIA TÉRMICA}

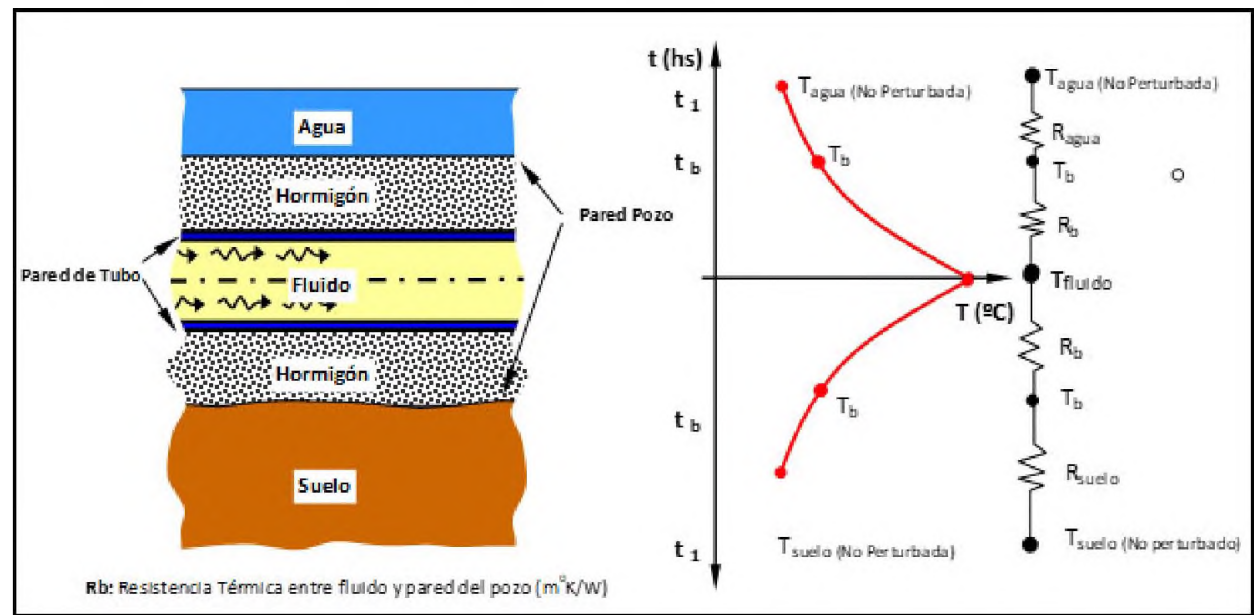

Figura 4: Esquema de resistencia témica entre el fluido y la pared del pozo $\mathrm{Rb}\left({ }^{\circ} \mathrm{K}\right.$ / (W i $\mathrm{m}$ )).

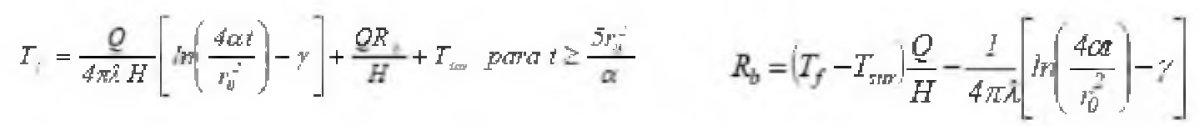

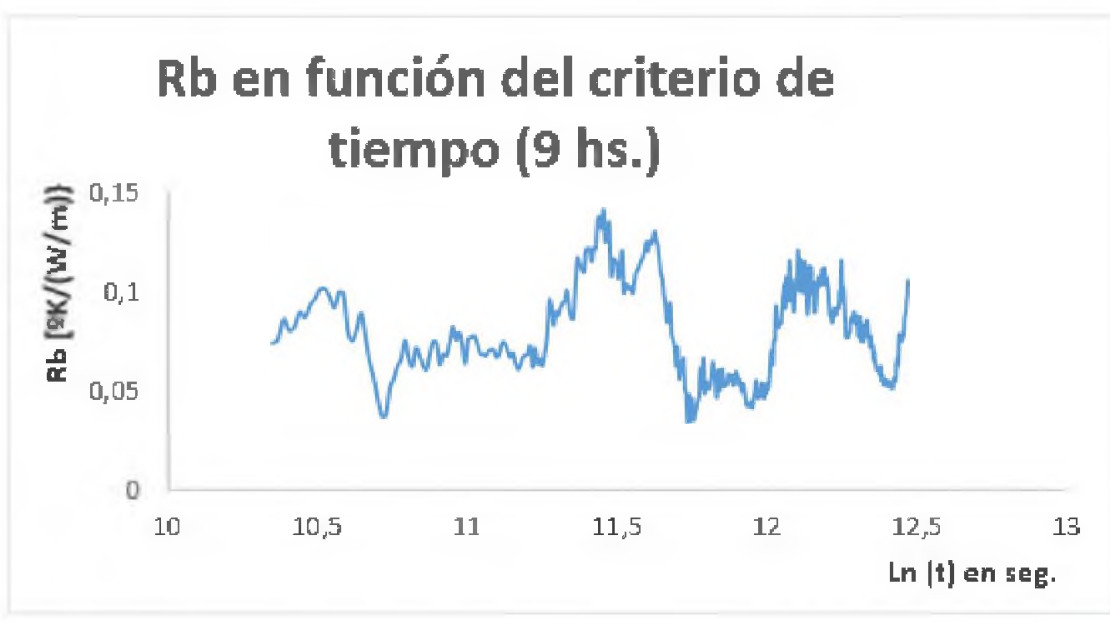

Figura 5: Rb en función de tiempo, aplicando criterio de tiempo 9 hs.

El valor promedio para $\mathrm{Rb}$ para la duración total del ensayo es de: $0,117\left[{ }^{\circ} \mathrm{K} /(\mathrm{W} / \mathrm{m})\right]$.

El valor promedio para $\mathrm{Rb}$ aplicando el criterio de tiempo es de:0,087 $\left[{ }^{\circ} \mathrm{K} /(\mathrm{W} / \mathrm{m})\right]$. 


\section{AJUSTE PARAMÉTRICO DE DATOS EXPERIMENTALES}

Se ha utilizado, como función de ajuste, la solución al modelo matemático que representa el proceso de transferencia de calor, con $\lambda$ eff y $\mathrm{Rb}$ obtenidos experimentalmente como variables de ajuste, figura 7. En ella es posible determinar la evolución de $\operatorname{Tf}(\mathrm{t})$ en función del $\operatorname{Ln}(\mathrm{t})$ en hs. curva azul. A ésta se le superpone la curva de ajuste paramétrico de los datos experimentales, curva roja, donde se observa buen ajuste y bajo error medio cuadrático acumulado.

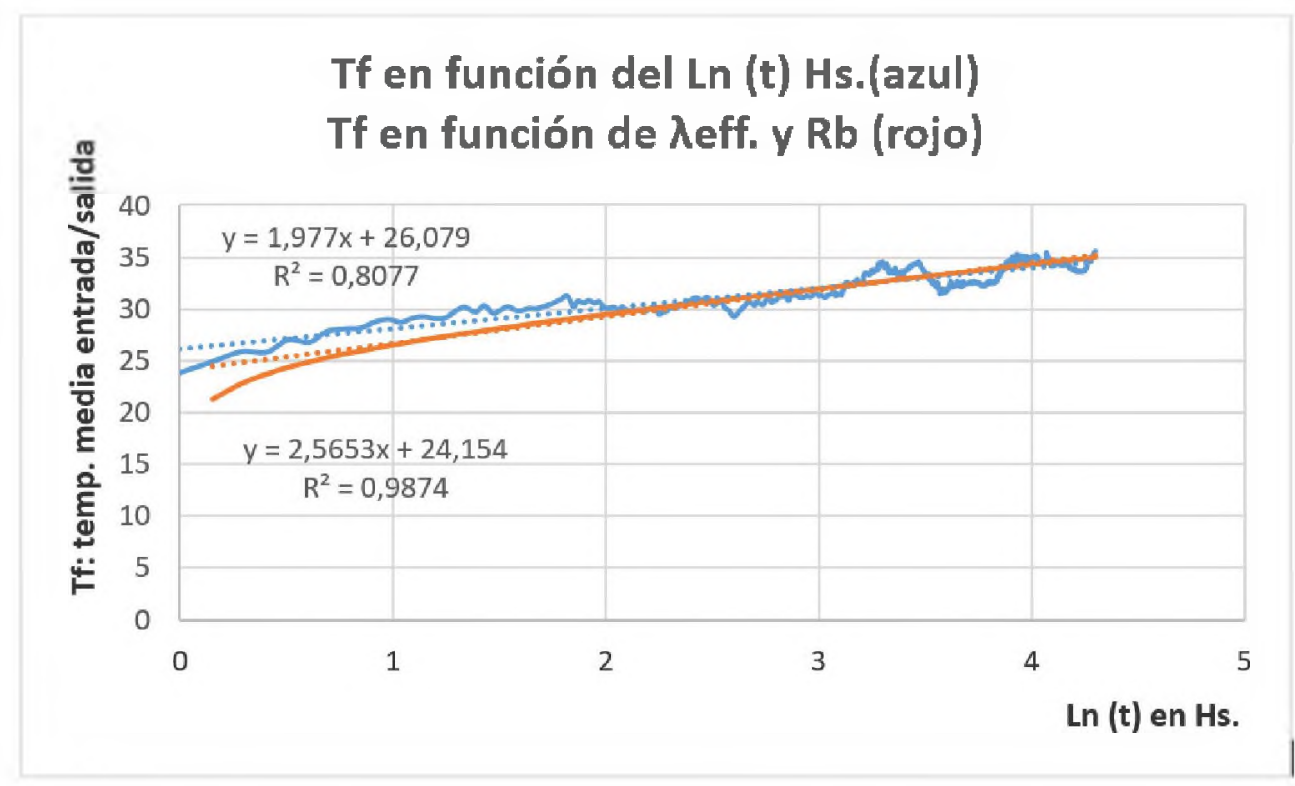

Figura 6: Evolución de la temperatura media del fluido (curva azul) y superpuesta la curva de ajuste paramétrico (curva roja)

\section{DISCUSION}

\begin{tabular}{|c|c|c|c|c|}
\hline $\begin{array}{l}\alpha: \text { Difusividad térmica del } \\
\text { hormigón }(\mathrm{m} 2 / \mathrm{seg})\end{array}$ & Criterio tiempo & Pendiente: $\mathrm{K}$ & $\lambda$ eff $\left(\mathrm{W} / \mathrm{m}^{* 0} \mathrm{~K}\right)$ & $\mathrm{Rb}:(\mathrm{K} /(\mathrm{W} / \mathrm{m})$ \\
\hline $0,91 \times 10-6$ & Total & 1,97 & 1,42 & 0,117 \\
\hline $0,91 \times 10-6$ & $9 \mathrm{hs}$ & 2,28 & 1,23 & 0,087 \\
\hline
\end{tabular}

Tabla 1: Resultados del segundo test de respuesta térmica San Luis - Argentina 


\section{RECOPILACIÓN DE TRT MUNDIALES}

\begin{tabular}{|c|c|c|c|c|}
\hline Borehole & TRT-unit & | (XI.) & 2 (DF. UBe(i) & 3 (DE) \\
\hline \multicolumn{2}{|c|}{ 1, backfilled with Mol-sand } & $i=2.47 W(\mathrm{~m} \cdot \mathrm{K})$ & - & $i=2.47 \mathrm{Wi}(\mathrm{m} \mathrm{K})$ \\
\hline \multicolumn{2}{|c|}{ 2, backfilled with graded sand } & $\lambda=2.40 \mathrm{~W} /(\mathrm{m} \cdot \mathrm{k})$ & - & $i=2.51 \mathrm{~W}(\mathrm{mK})$ \\
\hline \multicolumn{2}{|c|}{ 3. backfilled with bentonite } & test disturbed & $\lambda_{0}=2.49 \mathrm{~W}:(\mathrm{m} \mathrm{k})$ & - \\
\hline \multicolumn{5}{|c|}{ 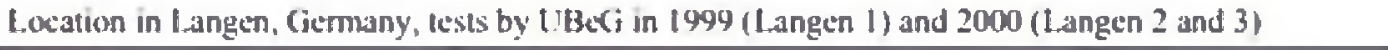 } \\
\hline Borchole & Depth & Girout & themal conductivity & borchole therm res. \\
\hline I.angen 1 & $99 \mathrm{~m}$ & standard bentonite & $\lambda=2.8 \mathrm{~W} /(\mathrm{m} \cdot \mathrm{K})$ & $\mathrm{r}_{\mathrm{b}}-0.11 \mathrm{~K}(\mathrm{~W} \cdot \mathrm{m})$ \\
\hline L.angen 2 & $70 \mathrm{~m}$ & therm enhanced & $\lambda=2.3 \mathrm{~W} /(\mathrm{m} \mathrm{k})$ & $\mathrm{r}_{\mathrm{b}}=0.08 \mathrm{~K}(\mathrm{~W} \cdot \mathrm{m})$ \\
\hline Langen 3 & $70 \mathrm{~m}$ & therm, enhanced & $\lambda=2.2 \mathrm{~W} /(\mathrm{m}-\mathrm{K})$ & $\mathrm{r}_{\mathrm{b}}-0.07 \mathrm{~K}(\mathrm{~W}-\mathrm{m})$ \\
\hline \multicolumn{5}{|c|}{ Location in Maine, Germany, tesis by UBex; in summer 2003} \\
\hline Matinz I & $30 \mathrm{~m}$ & standard & $\lambda=1.43 W(\mathrm{~m} \cdot \mathrm{k})$ & $r_{b}-0.16 \mathrm{~K}(\mathrm{~W} \cdot \mathrm{m})$ \\
\hline Mainz 2 & $30 \mathrm{~m}$ & standard & $\lambda=1 .+1 W(\mathrm{~m} \cdot \mathrm{k})$ & $\mathrm{T}_{\mathrm{b}}-0.20 \mathrm{~K}(\mathrm{~W} \cdot \mathrm{m})$ \\
\hline
\end{tabular}

Tabla2: Results of multiple TRT on the site (Mol TRT workshop in 2000 and projects in Germany)

\section{CONCLUSIONES}

La idea principal de este trabajo es poder determinar tanto conductividad efectiva $\lambda$ eff $\left(\mathrm{W} / \mathrm{m}^{*} \mathrm{~K}\right)$ como la resistencia térmica del suelo $\mathrm{Rb}(\mathrm{K} /(\mathrm{W} / \mathrm{m})$, para un intercambiador amoldado a condiciones preexistentes, ubicado provincia de San Luis, Argentina.

La directriz VDI 4640 "Thermal use of the underground", para instalaciones con una potencia térmica de hasta $30 \mathrm{~kW}$, señala reglas de dimensionamiento, con valores de $\lambda$ eff y Rb. dados por esta directriz mientras que para potencias térmicas mayores exige la realización del TRT, de esta forma se logra el correcto dimensionado del captador al poder obtener in situ los valores reales de $\lambda$ y Rb. los cuales constituyen el elemento clave para este tipo de instalaciones geotérmicas, porque se traduce en costos.

Como es posible detectar en este traba- jo, la conductividad térmica efectiva $\lambda$ eff, está dada por la pendiente de la curva determinada por la nube de puntos de las mediciones de temperatura media de entrada y salida respecto al logaritmo natural del tiempo. El valor de esta pendiente está determinado por el criterio de tiempo empleado y a su vez este valor está relacionado con la difusividad térmica del sistema (hormigón), que se adopta por medio de tablas.

En este caso en particular, se obtiene un $\lambda$ eff, que sería el reflejo del sistema en cuestión, ya que, por un lado, se presenta un suelo sin rocas y sobre el otro, agua.

Comparando datos entre el ensayo realizado en San Luis, con otros ensayos (tabulados ut-supra), es posible afirmar que el método grafico de la pendiente, utilizado en este caso para obtener los valores de $\lambda$ eff. y $\mathrm{Rb}$, es suficientemente aproximado. 
Observaciones sobre el ensayo realizado, el método de adquisición de datos fue manual, el suministro de potencia de inyección fue bastante variable durante el mismo y la temperatura de suelo no perturbada es un poco elevada.

\section{REFERENCIAS}

Austin III, W. A., (1998), Development of an Situ System for Measuring Ground Termal Properties. Tesis de Maestria, Oklahoma State University.

Busso A., Reuss M., Müller J., Sograri N. (2000), Almacenamiento Subterráneo de Energía Térmica: Resultado de un ensayo de respuestas térmicas del subsuelo. Avance en Energías Renovables y Medio Ambiente, Vol. 4, No 1.

Busso A., Reuss M., (2000), Ensayo de Respuestas Térmicas: Metodo de Evaluación de la Pendiente vs Ajuste Con Dos Parámetros Variables. Dpto de Física - Facultad de Ciencias Exactas y Naturales, UNNE - Corrientes Argentina.

Eklöf C, Gehlin S. (1996). A Mobile Equipment for Thermal Response Test. Master of science thesis 1996:198E. LuleåUniversity of Technology. Sweden.

Gehlin, S. and Nordell, B. (1997). "Thermal Response Test - a Mobile Equipment for Determining Thermal Resistance of Borehole." Proc. Megastock '97 Sapporo, pp. 103-108
Hellström, G. (1997). “Thermal response test of a heat store in clay at Linköping, Sweden." Proc. Megastock' 97 Sapporo, pp. 115-120

Ingersoll, L.R. and Plass, H.J. (1948). "Theory of the ground pipe heat source for the heat pump." Heating, Piping \& Air Conditioning 20/7, pp. 119-122

Páginas web:

http://ubeg.de/Lit/2016\%20Sauer\%20et\%20 al\%20egc\%202016.pdf 28/09/2018

\section{NOMENCLATURA}

$\operatorname{Tr}(t)$ : temperatura en el tiempo " $t$ ".

Tr: temperatura estacionaria originada por la temperatura media del fluido inyectado.

Tsur: temperatura de suelo no perturbado.

$\operatorname{Trq}(\mathrm{t})$ : cambio de temperatura debido a la desviación de la temperatura media del fluido.

Tf: Temperatura del fluido portador de calor $\left[{ }^{\circ} \mathrm{C}\right]$

Q: Potencia inyectada [W]

H: Profundidad eficaz del pozo [m]

$\mathrm{q}=\mathrm{Q} / \mathrm{H}$ : Potencia inyectada por metro de tubo $[\mathrm{W} / \mathrm{m}]$

$\lambda$ eff: Conductividad térmica del medio (W/m $\circ \mathrm{K})$

$\gamma: 0,5772$ (Const. de Euler)

$\mathrm{r} 0$ : Radio del pozo (m)

$\alpha$ : Difusividad térmica ( $\mathrm{m} 2 / \mathrm{seg}$ )

Rb: Resistencia térmica entre el fluido portador de calor y la pared del pozo $[\circ \mathrm{K}(\mathrm{W} / \mathrm{m})]$

t: Tiempo [seg]

tb: Intervalo de tiempo [seg]

k: Pendiente de la recta de regresión lineal 